\title{
COMMUTATIVE ALGEBRAS AND COHOMOLOGY
}

BY

\author{
D. K. HARRISON ${ }^{(1)}$
}

This paper has three purposes: (1) to develop the machinery of a commutative cohomology theory for commutative algebras, (2) to apply this cohomology theory to give a working theory of ring extensions for commutative algebras, and (3) to employ and test the theory by relating certain natural cohomological conditions to corresponding conditions in algebraic geometry.

The first purpose is approached in the first and third sections. Our cohomology has several properties not enjoyed by the Hochschild theory (an explicit relation for the tensor product, a workable sequence for changes from an algebra to a factor algebra, and an explicit formula for going local); however, unlike the Hochschild theory, we have no dimension shifting techniques. For this reason, we restrict consideration to the first, second, and third cohomology modules.

The second purpose is considered in the second section. For $B$ and $A$ commutative algebras, we let $M(A)$ denote $\operatorname{Hom}_{A}(A, A)$, taken modulo the ideal of multiplications by elements in $A$. Following the prototype theory which Eilenberg and Mac Lane developed for groups, we associate to each algebra homomorphism from $B$ into $M(A)$, an element in a third cohomology module. The homomorphism is called unobstructed if this element is zero. The unobstructed homomorphisms, together with the elements of a certain second cohomology module, are associated in a one-one fashion with the commutative algebra extensions of $A$ by $B$.

The purpose of the fourth and last section is best expressed by considering a point $p$ on an affine variety $V$ over a perfect field $k$. Let $A$ be the coordinate ring of $V, p$ be the prime ideal associated with $p$, and $Q$ be the quotient field of $A / \mathfrak{p}$. We prove that $p$ is a simple point if and only if the second cohomology module of $A$ with coefficients in $Q$ is zero. In this manner we give a characterization of regular local rings. We also prove that $p$ will be a complete intersection if and only if the difference between the dimensions of the first and second cohomology modules (both of $A$ with coefficients in $Q$ ) is the dimension of $V$. Our third main result is that $V$ will be nonsingular if and only if $A$ has trivial second cohomology with respect to all finitely generated $A$ modules.

Received by the editors February 25, 1961 and, in revised form, September 5, 1961.

(1) This paper consists of work developed by the author under the National Science Foundation contract G 9664 . 
1. Commutative cohomology. Let $k$ be a field and $A$ be a commutative associate algebra (possibly infinite dimensional) over $k$. Let $E$ be an $A$-module (this includes the assumption that $E$ is a vector space over $k$ ). Before defining specifically the three special cohomology modules which will actually concern us, we consider briefly the general commutative cohomology situation. Let $T$ be the tensor algebra (without the usual identity adjoined) of $A$. Then $T$ with the shuffle product (see [1]) is a skew-commutative, graded algebra. $\operatorname{Hom}\left(T / T^{2}, E\right)$ will turn out to be a complex with the usual coboundary operator $\left({ }^{2,3}\right)$. We denote the homology module of this complex at the $n$th place by $\mathcal{E}^{n}(A, E)$. Theorem 2 which follows and which only involves the cohomology modules for $n=1,2$, and 3 will be our main tool. It is because we can not extend this result to the higher modules (actually an extension exists but it involves unknown modules) that we limit ourselves in this paper to $n=1,2$, and 3 . We point out that a satisfactory extension of Theorem 2 together with our $\$ 2$ would lead, by induction on the index of nilpotency, to a complete theory for finite dimensional nilpotent commutative algebras (and thus for all finite dimensional commutative algebras).

The first cohomology group is the module of all derivations from $A$ to $E$. Let $Z^{2}(A, E)$ be the module of all symmetric bilinear maps $f$ from $A \times A$ to $E$ such that

$$
\alpha f(\beta, \gamma)-f(\alpha \beta, \gamma)+f(\alpha, \beta \gamma)-\gamma f(\alpha, \beta)=0
$$

for all $\alpha, \beta, \gamma \in A$. Let $B^{2}(A, E)$ be all those $f$ such that there is a linear map $g$ from $A$ to $E$ with

$$
f(\alpha, \beta)=\alpha g(\beta)-g(\alpha \beta)+\beta g(\alpha)
$$

for all $\alpha, \beta \in A$. Then $\varepsilon^{2}(A, E)=Z^{2}(A, E) / B^{2}(A, E)$. Finally let $Z^{3}(A, E)$ be all trilinear maps $f$ from $A \times A \times A$ to $E$ such that

$$
f(\alpha, \beta, \gamma)-f(\beta, \alpha, \gamma)+f(\beta, \gamma, \alpha)=0
$$

and

$$
\alpha f(\beta, \gamma, \delta)-f(\alpha \beta, \gamma, \delta)+f(\alpha, \beta \gamma, \delta)-f(\alpha, \beta, \gamma \delta)+\delta f(\alpha, \beta, \gamma)=0
$$

for all $\alpha, \beta, \gamma, \delta \in A$. If $B^{3}(A, E)$ denotes those $f$ such that there is a symmetric, bilinear $g$ from $A \times A$ to $E$ with

$$
f(\alpha, \beta, \gamma)=\alpha g(\beta, \gamma)-g(\alpha \beta, \gamma)+g(\alpha, \beta \gamma)-\gamma g(\alpha, \beta)
$$

for all $\alpha, \beta, \gamma \in A$, then $\mathcal{E}^{3}(A, E)=Z^{3}(A, E) / B^{3}(A, E)$. Following usual arguments we get

(2) The author is indebted to the referee for this simple definition of our commutative cohomology modules. The original equivalent definition was given in terms of complicated permutations which were rigged up so that an extension of Theorem 2 would hold.

(3) See, say [2], for the usual coboundary map. 
Theorem 1. If $L$ is a submodule of an $A$-module $E$, then there is a natural exact sequence

$$
0 \rightarrow \mathcal{E}^{1}(A, L) \rightarrow \mathcal{E}^{1}(A, E) \rightarrow \mathcal{E}^{1}(A, E / L) \rightarrow \mathcal{E}^{2}(A, L) \rightarrow \mathcal{E}^{2}(A, E) \rightarrow \cdots
$$

Either direct computations or the usual arguments for complexes can be used to give a long but straightforward proof of our next result. $A^{*}$ will denote $A$ if $E$ is unitary (that is, if $A$ has an identity 1 with $1 \cdot e=e$ for all $e \in E)$; otherwise, $A^{*}$ will denote $A$ with an identity adjoined. It is necessary to point out that

$$
\operatorname{Ext}_{A^{*}}^{1}(\mathfrak{a}, E) \simeq H^{1}\left(A^{*}, \operatorname{Hom}_{k}(\mathfrak{a}, E)\right) \simeq H^{1}\left(A, \operatorname{Hom}_{k}(\mathfrak{a}, E)\right)
$$

(see p. 170 of [3] and see [4]).

Theorem 2. If $\mathfrak{a}$ is an ideal in $A$ with $\mathfrak{a} \cdot E=0$, then there is a natural exact sequence

$$
\begin{aligned}
0 & \rightarrow \varepsilon^{1}(A / \mathfrak{a}, E) \rightarrow \varepsilon^{1}(A, E) \rightarrow \operatorname{Hom}_{A^{*}}(\mathfrak{a}, E) \\
& \rightarrow \varepsilon^{2}(A / \mathfrak{a}, E) \rightarrow \mathcal{E}^{2}(A, E) \rightarrow \mathfrak{e}^{1}(A, \mathfrak{a}, E) \\
& \rightarrow \varepsilon^{3}(A / \mathfrak{a}, E) \rightarrow \mathcal{E}^{3}(A, E)
\end{aligned}
$$

where $\mathfrak{C}^{1}(A, \mathfrak{a}, E)$ is a submodule of $\operatorname{Ext}_{A}^{1}{ }^{*}(\mathfrak{a}, E)$.

The next two lemmas follow from tedious repeated applications of the defining conditions for $Z^{2}(A, E)$ and $Z^{3}(A, E)$.

Lemma 3. If $f \in Z^{2}(A, E)$ then $f\left(\alpha \alpha^{\prime}, \beta \beta^{\prime}\right)=\alpha \beta f\left(\alpha^{\prime}, \beta^{\prime}\right)+\alpha^{\prime} \beta^{\prime} f(\alpha, \beta)-\alpha \alpha^{\prime} f\left(\beta, \beta^{\prime}\right)+f\left(\alpha \beta, \alpha^{\prime} \beta^{\prime}\right)-\beta \beta^{\prime} f\left(\alpha, \alpha^{\prime}\right)$ for all $\alpha, \alpha^{\prime}, \beta, \beta^{\prime} \in A$.

Lemma 4. If $f \in Z^{3}(A, E)$ then

$$
\begin{aligned}
f\left(\alpha \alpha^{\prime}, \beta \beta^{\prime}, \gamma \gamma^{\prime}\right)= & \alpha \beta \gamma f\left(\alpha^{\prime}, \beta^{\prime}, \gamma^{\prime}\right)+\alpha^{\prime} \beta^{\prime} \gamma^{\prime} f(\alpha, \beta, \gamma) \\
& -\alpha \alpha^{\prime}\left[f\left(\beta, \beta^{\prime}, \gamma \gamma^{\prime}\right)+\beta f\left(\gamma, \gamma^{\prime}, \beta^{\prime}\right)-f\left(\beta, \gamma, \beta^{\prime} \gamma^{\prime}\right)\right] \\
& +\left[f\left(\alpha \beta, \alpha^{\prime} \beta^{\prime}, \gamma \gamma^{\prime}\right)+\alpha \beta f\left(\gamma, \gamma^{\prime}, \alpha^{\prime} \beta^{\prime}\right)-f\left(\alpha \beta, \gamma, \alpha^{\prime} \beta^{\prime} \gamma^{\prime}\right)\right] \\
& -\left[f\left(\alpha, \alpha^{\prime}, \beta \gamma \beta^{\prime} \gamma^{\prime}\right)+\alpha f\left(\beta \gamma, \beta^{\prime} \gamma^{\prime}, \alpha^{\prime}\right)-f\left(\alpha, \beta \gamma, \alpha^{\prime} \beta^{\prime} \gamma^{\prime}\right)\right] \\
& +\gamma \gamma^{\prime}\left[f\left(\alpha, \alpha^{\prime}, \beta \beta^{\prime}\right)+\alpha f\left(\beta, \beta^{\prime}, \alpha^{\prime}\right)-f\left(\alpha, \beta, \alpha^{\prime} \beta^{\prime}\right)\right]
\end{aligned}
$$

for all $\alpha, \beta, \gamma, \alpha^{\prime}, \beta^{\prime}, \gamma^{\prime} \in A$.

Theorem 5. Let $A$ and $A^{\prime}$ be commutative algebras with identities. Let $E$ be both a unitary $A$-module and a unitary $A^{\prime}$-module with $\alpha\left(\alpha^{\prime} e\right)=\alpha^{\prime}(\alpha e)$, for all $\alpha \in A, \alpha^{\prime} \in A^{\prime}, e \in E$. Then

$$
\mathcal{E}^{n}\left(A \otimes A^{\prime}, E\right) \simeq \mathcal{E}^{n}(A, E) \oplus \mathcal{E}^{n}\left(A^{\prime}, E\right)
$$

for $n=1,2$, and 3 . 
Proof. For $n=1$, the result is easily computed. For $n=2, f \in Z^{2}(A, E)$, and $f^{\prime} \in Z^{2}\left(A^{\prime}, E\right)$ we map $f \oplus f^{\prime}$ onto $h$ where

$$
h\left(\alpha \otimes \alpha^{\prime}, \beta \otimes \beta^{\prime}\right)=\alpha \beta f^{\prime}\left(\alpha^{\prime}, \beta^{\prime}\right)+\alpha^{\prime} \beta^{\prime} f(\alpha, \beta)
$$

for all $\alpha, \beta \in A, \alpha^{\prime}, \beta^{\prime} \in A^{\prime}$. It is easily checked that this defines a monomorphism from $\mathcal{E}^{2}(A, E) \oplus \mathcal{E}^{2}\left(A^{\prime}, E\right)$ into $\mathcal{E}^{2}\left(A \otimes A^{\prime}, E\right)$. To show that this map is onto, we let $t+B^{2}\left(A \otimes A^{\prime}, E\right)$ be any element in $\mathcal{E}^{2}\left(A \otimes A^{\prime}, E\right)$ and employ Lemma 3 to get that

$$
\begin{aligned}
& t\left(\alpha \otimes \alpha^{\prime}, \beta \otimes \beta^{\prime}\right)=\alpha \beta t\left(1 \otimes \alpha^{\prime}, 1 \otimes \beta^{\prime}\right)+\alpha^{\prime} \beta^{\prime} t\left(\alpha \otimes 1^{\prime}, \beta \otimes 1^{\prime}\right) \\
& \quad-\alpha \alpha^{\prime} t\left(\beta \otimes 1^{\prime}, 1 \otimes \beta^{\prime}\right)+t\left(\alpha \beta \otimes 1^{\prime}, 1 \otimes \alpha^{\prime} \beta^{\prime}\right)-\beta \beta^{\prime} t\left(\alpha \otimes 1^{\prime}, 1 \otimes \alpha^{\prime}\right)
\end{aligned}
$$

for all $\alpha, \beta \in A, \alpha^{\prime}, \beta^{\prime} \in A^{\prime}$. The proof for $n=3$ can be given in a similar manner using Lemma 4 instead of Lemma 3 , and then showing that an appropriate map is symmetric.

It is useful for all of these computations to note that if $E$ is unitary, $f \in Z^{2}(A, E)$, and $h \in Z^{3}(A, E)$, then by defining

$$
\begin{aligned}
f^{\prime}(\alpha, \beta)= & -\alpha f(1, \beta)+f(1, \alpha \beta)-\beta f(1, \alpha)+f(\alpha, \beta), \\
h^{\prime}(\alpha, \beta, \gamma)= & -\alpha(h(1, \beta, \gamma)+h(1, \gamma, \beta))+(h(1, \alpha \beta, \gamma)+h(1, \gamma, \alpha \beta)) \\
& -(h(1, \alpha, \beta \gamma)+h(1, \beta \gamma, \alpha))+\gamma(h(1, \alpha, \beta)+h(1, \beta, \alpha))+h(\alpha, \beta, \gamma)
\end{aligned}
$$

for all $\alpha, \beta, \gamma \in A$, we can show that $f^{\prime}(\alpha, \beta)=0$ when either of $\alpha, \beta$ is 1 , $h^{\prime}(\alpha, \beta, \gamma)=0$ when any of $\alpha, \beta, \gamma$ is 1 , and $f-f^{\prime} \in B^{2}(A, E), h-h^{\prime} \in B^{3}(A, E)$.

Another remark which we shall need is that Theorem 5 is true for infinite tensor products. If $I$ is a linearly ordered indexing set and if we have algebras with identities, $A_{i}, i \in I$, then $\otimes A_{i}$ is the direct limit of the finite tensor products. If $E$ is a unitary $\otimes A_{i}$-module, then it is a straightforward extension of the proof of Theorem 5 , that

$$
\mathcal{E}^{n}\left(\otimes A_{i}, E\right) \simeq \prod \mathcal{E}^{n}\left(A_{i}, E\right)
$$

for $n=1,2,3$ where $\prod$ denotes the unrestricted direct sum.

2. Commutative algebra extensions. If $D$ is a commutative algebra and $\mathfrak{a}$ is an ideal, then $\mathfrak{a}$ and $D / \mathfrak{a}$ are algebras and $D$ is a commutative extension of these two algebras. Our aim is to start with $\mathfrak{a}$ and $D / \mathfrak{a}$ and to find all ways that they can be put together. The results are considerably simpler than if commutativity is not assumed (see [5]). Our methods are an extension to commutative algebras of the ideas of [6].

Let $A$ be a commutative algebra. Consider $\operatorname{Hom}_{A}(A, A)$; this is the set of all $k$ linear maps from $A$ to $A$ such that $f(\alpha \beta)=\alpha f(\beta)$ for all $\alpha, \beta \in A$. $\operatorname{Hom}_{A}(A, A)$ is an algebra in the obvious fashion. If $\alpha \in A$ we let $f_{\alpha} \in \operatorname{Hom}_{A}(A, A)$ be defined by $f_{\alpha}(\beta)=\alpha \beta$ for all $\beta \in A$. This gives an algebra homomorphism from $A$ to $\operatorname{Hom}_{A}(A, A)$ whose kernel is $0: A$ (the set of all $\alpha \in A$ such that $\alpha \beta=0$ for all $\beta \in A$ ). We denote the image by $\bar{A}$. It is easily 
checked to be an ideal in $\operatorname{Hom}_{A}(A, A)$. Let $M(A)$ be $\operatorname{Hom}_{A}(A, A) / \bar{A}$. For $g \in \operatorname{Hom}_{A}(A, A)$ we let $g^{\sharp}$ be $g+\bar{A}$ in $M(A)$. For $g^{\sharp} \in M(A), \alpha \in 0: A$ we define $g^{t} \cdot \alpha$ as $g(\alpha)$. It is easily checked that this makes $0: A$ into a left module over $M(A)$. In particular $0: A$ is a module over any commutative subalgebra of $M(A)$.

A subalgebra $W$ of $M(A)$ will be called strongly commutative if the subalgebra of $\operatorname{Hom}_{A}(A, A)$ consisting of all $g$ with $g^{*} \in W$ is commutative. Hence we have a one-one correspondence between the commutative subalgebras of $\operatorname{Hom}_{A}(A, A)$ which contain $\bar{A}$ and the strongly commutative subalgebras of $M(A)$. Incidentally it is easily checked that $\bar{A}$ is in the center of $\operatorname{Hom}_{A}(A, A)$.

Proposition 6. To each strongly commutative subalgebra $W$ of $M(A)$ we have associated in a natural fashion an element $\lambda_{W}$ of $\mathcal{E}^{3}(W, 0: A)$.

Proof. Choose arbitrarily a linear map $b \rightarrow g_{b}$ from $W$ to $\operatorname{Hom}_{A}(A, A)$ such that $g^{*}=b$ for all $b \in W$. Since $W$ is strongly commutative $g_{b_{1} b_{2}}-g_{b_{1}} \circ g_{b_{2}}$ gives a linear map from the symmetric product of $W$ with itself (those elements of $W \otimes W$ left fixed by $\bar{\sigma}$ where $\left.\bar{\sigma}\left(b_{1} \otimes b_{2}\right)=b_{2} \otimes b_{1}\right)$ into $\bar{A}$. Now arbitrarily pull this map back to a linear map from the symmetric product to $A$. Hence for $b_{1}, b_{2} \in W$ we have $\gamma\left(b_{1}, b_{2}\right) \in A$ with $\gamma\left(b_{1}, b_{2}\right)=\gamma\left(b_{2}, b_{1}\right)$ and $g_{b_{1} b_{2}}-g_{b_{1}} \circ g_{b_{2}}$ $=f_{\gamma\left(b_{1}, b_{2}\right)}$. For $b_{1}, b_{2}, b_{3} \in W$ let

$$
h\left(b_{1}, b_{2}, b_{3}\right)=g_{b_{1}}\left(\gamma\left(b_{2}, b_{3}\right)\right)-\gamma\left(b_{1} b_{2}, b_{3}\right)+\gamma\left(b_{1}, b_{2} b_{3}\right)-g_{b_{3}}\left(\gamma\left(b_{1}, b_{2}\right)\right) .
$$

It is tedious but straightforward to show that $h \in Z^{3}(W, 0: A)$. One must use that all the $g_{b}$ commute (since $W$ is strongly commutative), and that if $\alpha \in A$ then $\gamma\left(b_{1}, b_{2}\right) \alpha=g_{b_{1} b_{2}}(\alpha)-g_{b_{1}}\left(g_{b_{2}}(\alpha)\right)$. Let $\lambda_{W}$ be $h+B^{2}(W, 0: A)$. It is an easy computation to check that $\lambda_{W}$ is independent of the choice of the $\gamma\left(b_{1}, b_{2}\right)$. To show that it is independent of the choice of the $g_{b}$ is more difficult. Let $g^{\prime}$ be another choice. Then there is a linear map $b \rightarrow \alpha(b)$ from $W$ to $A$ with $g_{b}-g_{b}^{\prime}=f_{\alpha(b)}$ for all $b \in W$. Hence

$$
\begin{aligned}
f_{\gamma\left(b_{1}, b_{2}\right)} & =g_{b_{1} b_{2}}^{\prime}+f_{\alpha\left(b_{1} b_{2}\right)}-\left(g_{b_{1}}^{\prime}+f_{\alpha\left(b_{1}\right)}\right) \circ\left(g_{b_{2}}^{\prime}+f_{\alpha\left(b_{2}\right)}\right) \\
& =\left(g_{b_{1} b_{2}}^{\prime}-g_{b_{1}}^{\prime} \circ g_{b_{2}}^{\prime}\right)+f_{\alpha\left(b_{1}, b_{2}\right)}-f_{\alpha\left(b_{1}\right)} \circ f_{\alpha\left(b_{2}\right)}-g_{b_{1}}^{\prime} \circ f_{\alpha\left(b_{2}\right)}-g_{b_{2}}^{\prime} \circ f_{\alpha\left(b_{1}\right)} .
\end{aligned}
$$

This means that we can choose $\gamma^{\prime}\left(b_{1}, b_{2}\right)$ as

$$
\gamma\left(b_{1}, b_{2}\right)+\alpha\left(b_{1}\right) \cdot \alpha\left(b_{2}\right)-\alpha\left(b_{1} b_{2}\right)+g_{b_{1}}^{\prime}\left(\alpha\left(b_{2}\right)\right)+g_{b_{2}}^{\prime}\left(\alpha\left(b_{1}\right)\right) .
$$

If we use this and that $g_{b}^{\prime}=g_{b}-f_{\alpha(b)}$ to get rid of all prime terms in the definition of $h^{\prime}\left(b_{1}, b_{2}, b_{3}\right)$, we get after a long computation that $h^{\prime}\left(b_{1}, b_{2}, b_{3}\right)=h\left(b_{1}, b_{2}, b_{3}\right)$. This completes the proof.

Let $A$ and $B$ be commutative algebras and let $\Delta$ be an algebra homomorphism from $B$ to $M(A)$. $\Delta$ will be called unobstructed if :

(1) the image $W$ of $\Delta$ is strongly commutative,

(2) $\Delta^{\prime}\left(\lambda_{W}\right)=0$ where $\Delta^{\prime}$ is the natural map from $\mathcal{E}^{3}(W, 0: A)$ to $\mathcal{E}^{3}(B, 0: A)$ (since $\Delta$ is onto $W, 0: A$ is a $B$-module in a natural fashion). 
We remark that if $f \in Z^{3}(W, 0: A)$, then $\Delta^{\prime}\left(f+B^{3}(W, 0: A)\right)$ is the coset containing $f^{\prime}$ where $f^{\prime}\left(\beta_{1}, \beta_{2}, \beta_{3}\right)=f\left(\Delta\left(\beta_{1}\right), \Delta\left(\beta_{2}\right), \Delta\left(\beta_{3}\right)\right)$ for all $\beta_{1}, \beta_{2}, \beta_{3} \in B$. $\Delta^{\prime}$ is the map appearing in the exact sequence of Theorem 2 ,

$$
\cdots \rightarrow \mathcal{E}^{2}(B, 0: A) \rightarrow \mathfrak{e}^{1}(B, \mathfrak{b}, 0: A) \rightarrow \mathcal{E}^{3}(W, 0: A) \rightarrow \mathcal{E}^{3}(B, 0: A) \rightarrow \cdots
$$

where $\mathfrak{b}$ is the kernel of $\Delta$.

We return now to the problem of finding all extensions of a commutative algebra $A$ by a commutative algebra $B$. By an extension of $A$ by $B$ we mean a triple $(\phi, D, \theta)$ where $D$ is a commutative algebra, $\phi$ is a one-one algebra homomorphism from $A$ into $D, \theta$ is an algebra homomorphism from $D$ onto $B$, and the image of $\phi$ is the kernal of $\theta$. Thus except for isomorphisms, $A$ is an ideal of $D$ with $(D / A) \simeq B$. Two extensions $(\phi, D, \theta)$ and $\left(\phi^{\prime}, D^{\prime}, \theta^{\prime}\right)$ are called equivalent if there exists an isomorphism $g$ from $D$ to $D^{\prime}$ with $g \circ \phi=\phi^{\prime}$ and $\theta^{\prime} \circ g=\theta$. Theorems 7 and 8 will give computational characterization of the set of all equivalence classes of extension of $A$ by $B$.

Let $(\phi, D, \theta)$ be an extension of $A$ by $B$. For $\beta \in B$ we let $\Delta(\beta)=f+\bar{A}$ where $f(\alpha)=\phi^{-1}(\gamma \phi(\alpha))$ for all $\alpha \in A$ and where $\gamma$ is any element in $D$ with $\theta(\gamma)=\beta$. It is easy to check that $\Delta$ is a well defined algebra homomorphism from $B$ to $M(A)$. It is also easy to check that equivalent extensions give the same homomorphism.

Theorem 7. Let $A$ and $B$ be commutative algebras. An algebra homomorphism $\Delta$ from $B$ to $M(A)$ is the homomorphism associated with some extension of $A$ by $B$ if and only if it is unobstructed.

Proof. Let $\Delta$ be associated with $(\phi, D, \theta)$. For $\delta \in D$ let $i_{\delta}(\alpha)=\phi^{-1}(\delta \phi(\alpha))$ for all $\alpha \in A$. Note that $i_{\phi(\alpha)}=f_{\alpha}$ for $\alpha \in A$. Let $\mathfrak{b}$ be the kernel of $\Delta$. Then $\phi^{-1}(\mathfrak{b})=\left\{\delta \in D \mid i_{\delta}=i_{\phi(\alpha)}\right.$ for some $\left.\phi(\alpha) \in \phi(A)\right\}=\phi(A)+0: \phi(A)$. Let $t$ be a linear map from $\mathfrak{b}$ to $0: \phi(A)$ such that $\theta \circ t$ is the identity map. Extend $t$ to a map from $B$ to $D$ with $\theta \circ t$ the identity. For $\beta \in B, \Delta(\beta)=i_{t(\beta)}+\bar{A}$. Since $i_{t(\beta)}=0$ if $\beta \in \mathfrak{b}$, we may define $g_{b}$ as $i_{t\left(\Delta^{-1}(b)\right)}$ for $b \in W$ (the image of $\Delta$ ). We use the notation of the proof of Proposition 6. Since $D$ is commutative, the $g_{b}$ commute with each other and $W$ is strongly commutative. Let $s$ be a linear map from $W$ to $B$ with $\Delta \circ s$ the identity map. For $b_{1}, b_{2} \in W, s\left(b_{1} b_{2}\right)-s\left(b_{1}\right) \cdot s\left(b_{2}\right)$ $\in \mathfrak{b}$ ( $\Delta$ of it is zero) and thus $i_{t\left(s\left(b_{1} \cdot b_{2}\right)\right)}=i_{t\left(s\left(b_{1}\right) \cdot s(b(2))\right.}$. This means that we can choose $y\left(b_{1}, b_{2}\right)$ as $\phi^{-1}$ of $t\left(s\left(b_{1}\right) \cdot s\left(b_{2}\right)\right)-t\left(s\left(b_{1}\right)\right) \cdot t\left(s\left(b_{2}\right)\right)$. This is in $\phi(A)$ since $\theta$ of it is zero. Now for $\beta_{1}, \beta_{2} \in B$ we let

$r\left(\beta_{1}, \quad \beta_{2}\right)=t\left(s\left(\Delta\left(\beta_{1}\right)\right) \cdot s\left(\Delta\left(\beta_{2}\right)\right)\right)-t\left(\beta_{1} \cdot \beta_{2}\right)-t\left(s\left(\Delta\left(\beta_{1}\right)\right)\right) \cdot t\left(s\left(\Delta\left(\beta_{2}\right)\right)\right)+t\left(\beta_{1}\right) \cdot t\left(\beta_{2}\right)$.

Note that

$$
s\left(\Delta\left(\beta_{1}\right)\right) \cdot s\left(\Delta\left(\beta_{2}\right)\right)-s\left(\Delta\left(\beta_{1} \cdot \beta_{2}\right)\right)+s\left(\Delta\left(\beta_{1} \cdot \beta_{2}\right)\right)-\beta_{1} \cdot \beta_{2}
$$

is in $\mathfrak{b}$, as are $s\left(\Delta\left(\beta_{1}\right)\right)-\beta_{1}$ and $s\left(\Delta\left(\beta_{2}\right)\right)-\beta_{2}$. This can be used to show (since 
$t(\mathfrak{b}) \subset 0: \phi(A))$ that $r\left(\beta_{1}, \beta_{2}\right) \in 0: \phi(A)$. By taking $\theta$ of $r\left(\beta_{1}, \beta_{2}\right)$ we can see that it lies in $\phi(A)$. It is now an easy though tedious exercise to show that $h \circ(\Delta \times \Delta \times \Delta)$ can be computed from $\phi^{-1} \circ r$ in the way that makes $h \circ(\Delta \times \Delta \times \Delta) \in B^{3}(B, 0: A)$. Note for use in the next theorem that every element in $D$ can be written uniquely as $t(\beta)+\phi(\alpha)$ for $\beta \in B, \alpha \in A$, and then

$$
\begin{aligned}
& {[t(\beta)+\phi(\alpha)]+\left[t\left(\beta^{\prime}\right)+\phi\left(\alpha^{\prime}\right)\right]=t\left(\beta+\beta^{\prime}\right)+\phi\left(\alpha+\alpha^{\prime}\right)} \\
& {[t(\beta)+\phi(\alpha)] \cdot\left[t\left(\beta^{\prime}\right)+\phi\left(\alpha^{\prime}\right)\right]} \\
& =t\left(\beta \beta^{\prime}\right)+\phi\left(g_{\Delta(\beta)}\left(\alpha^{\prime}\right)+g_{\Delta\left(\beta^{\prime}\right)}(\alpha)+\alpha \alpha^{\prime}+\lambda\left(\beta, \beta^{\prime}\right)\right)
\end{aligned}
$$

where $\lambda\left(\beta, \beta^{\prime}\right)=\phi^{-1}\left(t(\beta) t\left(\beta^{\prime}\right)-t\left(\beta \beta^{\prime}\right)\right)$.

Now let $\Delta$ be an unobstructed homomorphism from $B$ to $M(A)$. Then there exists a symmetric bilinear map $r$ from $B$ to $0: A$ with

$\Delta\left(\beta_{1}\right) r\left(\beta_{2}, \beta_{3}\right)-r\left(\beta_{1} \beta_{2}, \beta_{3}\right)+r\left(\beta_{1}, \beta_{2} \beta_{3}\right)-\Delta\left(\beta_{3}\right) r\left(\beta_{1}, \beta_{2}\right)=h\left(\Delta\left(\beta_{1}\right), \Delta\left(\beta_{2}\right), \Delta\left(\beta_{3}\right)\right)$

for all $\beta_{1}, \beta_{2}, \beta_{3} \in B$. Let $\lambda\left(\beta_{1}, \beta_{2}\right)=\gamma\left(\Delta\left(\beta_{1}\right), \Delta\left(\beta_{2}\right)\right)-r\left(\beta_{1}, \beta_{2}\right)$ for $\beta_{1}, \beta_{2} \in B$. Let $D$ be all pairs $(\beta, \alpha)$ with $\beta \in B, \alpha \in A$. Define

$$
\begin{aligned}
(\beta, \alpha)+\left(\beta^{\prime}, \alpha^{\prime}\right) & =\left(\beta+\beta^{\prime}, \alpha+\alpha^{\prime}\right) \\
(\beta, \alpha) \cdot\left(\beta^{\prime}, \alpha^{\prime}\right) & =\left(\beta \beta^{\prime}, g_{\Delta(\beta)}\left(\alpha^{\prime}\right)+g_{\Delta\left(\beta^{\prime}\right)}(\alpha)+\alpha \alpha^{\prime}+\lambda\left(\beta, \beta^{\prime}\right)\right) .
\end{aligned}
$$

Let $\phi(\alpha)=(0, \alpha)$ and $\theta((\beta, \alpha))=\beta$. Then it is easily checked that $D$ is a commutative algebra and that $(\phi, D, \theta)$ is an extension of $A$ by $B$ whose homomorphism is $\Delta$.

THEOREM 8. Let $\Delta$ be an unobstructed homomorphism from $B$ to $M(A)$. Then the equivalence classes of extensions of $A$ by $B$ which have $\Delta$ for homomorphism are in one-one correspondence with the elements of $\mathcal{E}^{2}(B, 0: A)$.

Proof. As in the proof of Theorem 7 we choose an extension where $D$ is $B \oplus A$ and

$$
(\beta, \alpha) \cdot\left(\beta^{\prime}, \alpha^{\prime}\right)=\left(\beta \beta^{\prime}, g_{\Delta(\beta)}\left(\alpha^{\prime}\right)+g_{\Delta\left(\beta^{\prime}\right)}(\alpha)+\alpha \alpha^{\prime}+\lambda\left(\beta, \beta^{\prime}\right)\right) .
$$

We had noted that all extensions are like this except possibly with a different map $\lambda^{\prime}\left(\beta, \beta^{\prime}\right)$ in place of $\lambda\left(\beta, \beta^{\prime}\right)$. It is easy to check that $\lambda^{\prime}$ must be and can be of the form $\lambda+s$ where $s \in Z^{2}(B, 0: A)$. For $s, s^{\prime} \in Z^{2}(B, 0: A)$ the extensions for $\lambda+s$ and $\lambda+s^{\prime}$ will be equivalent if and only if they give the same element in $\mathcal{E}^{2}(B, 0: A)$.

We close this section with some remarks about $M(A)$. Clearly $M(A)=0$ if and only if $A$ has an identity. $M(A)=k$ is the next simplest situation.

Proposition 9. If $A$ is nilpotent, then $M(A)=k$ if and only if $A^{*}$ ( $A$ with an identity) is self-injective (which is equivalent to $A^{*}$ being quasi-Frobenious if $\operatorname{dim} A$ is finite).

Proof. Using that $A$ is nilpotent, it is easily checked that $\operatorname{Hom}_{A^{*}}\left(A, A^{*}\right)$ 
$=\operatorname{Hom}_{A^{*}}(A, A)$. Hence the exact sequence

$$
0 \rightarrow A \rightarrow A^{*} \rightarrow k \rightarrow 0
$$

gives

$$
\operatorname{Hom}_{A^{*}}\left(A^{*}, A^{*}\right) \rightarrow \operatorname{Hom}_{A^{*}}\left(A, A^{*}\right) \rightarrow \operatorname{Ext}_{A^{*}}^{1}\left(k, A^{*}\right) \rightarrow 0
$$

and thus $\operatorname{Ext}_{A^{*}}^{1}\left(k, A^{*}\right) \simeq M(A) / k$. If $\operatorname{Ext}_{A^{*}}^{1 *}\left(k, A^{*}\right)=0$ and $E$ is any $A^{*}$ module, then by the exact sequence

$$
0 \rightarrow N \cdot E \rightarrow E \rightarrow E / N \cdot E \rightarrow 0
$$

and induction on the smallest $n$ with $N^{n} \cdot E=0$, we get $\operatorname{Ext}_{A^{*}}^{1 *}\left(E, A^{*}\right)=0$. For the parenthetical remark we refer to [7].

Proposition 10. If $A^{*}$ is an integrally closed Noetherian domain, then $M(A)=k$.

Proof. Let $F$ be the quotient field of $A^{*}$. For $\alpha \neq 0, \beta \neq 0$ elements of $A$, $\alpha f(\beta)=f(\alpha \beta)=\beta f(\alpha)$ so $f(\beta) / \beta=f(\alpha) / \alpha$. But $f(\beta) / \beta$ times the ideal $A$ is in $A$ and thus by the usual determinant argument for integrally closed domains, $f(\beta) / \beta \in A^{*}$ (see $[8]$ ).

3. Calculations for $\varepsilon^{2}$. In this section we give some computational theorems for $\mathcal{E}^{2}$. Perhaps our most interesting result is a canonical characterization for polynomial rings.

Let $k$ be a field and $X$ be a set of indeterminants. Let $R$ be $k[X]$ and $R_{n}$ be the subspace of $R$ generated by the monomials of degree $n$. Then $R$ is a graded algebra $\left(R=\sum R_{n}\right.$ direct and $\left.R_{n} R_{m} \subset R_{n+m}\right)$ and it is connected (this terminology comes from homology rings and means that $R_{0}=k 1$ ). Also $R_{n}=\left(R_{1}\right)^{n}$ for all $n$. We remark that if $A=\sum_{n>0} R_{n}$, then $E$ will be an $A$ module if and only if it is a unitary $R$-module; also, $\varepsilon^{n}(R, E)=\varepsilon^{n}(A, E)$ for $n=1,2,3$.

THEOREM 11. If $R$ is a polynomial ring and $E$ is any unitary $R$-module, then $\mathcal{E}^{2}(R, E)=0$ and $\mathcal{E}^{3}(R, E)=0$. Conversely if $R=\sum R_{n}$ is a connected graded algebra with $\left(R_{1}\right)^{n}=R_{n}$ for all $n$ and $\varepsilon^{2}(R, k)=0$, then $R$ is a polynomial ring.

Proof. The first part follows from the two remarks after Theorem 5 together with the next three lemmas. We use that any polynomial ring is a tensor product of copies of $k[x]$.

Lemma 12. If $f \in Z^{2}(k[x], E)$ with $f\left(1, x^{n}\right)=0$ for all $n$, then $f\left(x^{m}, x^{n}\right)$ $=-x^{m} g\left(x^{n}\right)+g\left(x^{m+n}\right)-x^{n} g\left(x^{m}\right)$ for all $m, n$ where $g\left(x^{m}\right)=\sum x^{i} f\left(x, x^{m-1-i}\right)$, with the sum from $i=0$ to $i=m-1$.

Proof. Induction on $m$.

Lemma 13. If $f \in Z^{2}(k[x], E)$ with $f\left(1, x^{n}, x^{s}\right)=0$ for all $n$, $s$, then 


$$
f\left(x^{m}, x^{n}, x^{s}\right)=-x^{m} h\left(x^{n}, x^{s}\right)+h\left(x^{m+n}, x^{s}\right)-h\left(x^{m}, x^{n+s}\right)+x^{s} h\left(x^{m}, x^{n}\right)
$$

for all $m, n, s$ where

$$
h\left(x^{m}, x^{n}\right)=\sum x^{i} f\left(x, x^{m-1-i}, x^{n}\right),
$$

with the sum from $i=0$ to $i=m-1$.

Proof. Induction on $m$.

Lemma 14. With $h$ defined as in the last lemma, $h\left(x^{m}, x^{n}\right)=h\left(x^{n}, x^{m}\right)$ for all $m, n$.

Proof.

$$
\begin{aligned}
h\left(x^{m}, x^{n}\right) & =\sum x^{i} f\left(x, x^{m-1-i}, x^{n}\right) \\
& =\sum x^{i} f\left(x, x^{n}, x^{m-1-i}\right)-\sum x^{i} f\left(x^{n}, x, x^{m-1-i}\right) .
\end{aligned}
$$

If we substitute into this the formula of Lemma 13, simplify, and use that $h\left(x, x^{s}\right)=0$ for all $s$, we get $h\left(x^{n}, x^{m}\right)$.

We now must prove the second part of Theorem 11. Let $X$ be a set of indeterminants in one-one correspondence with a basis of $R_{1}$. We have a homomorphism from $k[X]$ onto $R$ with kernel $\mathfrak{b} . \mathfrak{b} \subset \mathfrak{a}^{2}$ where $\mathfrak{a}$ is the ideal in $k[X]$ generated by the set $X$. If $f \in \mathcal{E}^{1}(k[X], k)$ and $\alpha, \beta \in \mathfrak{a}$, then $f(\alpha \beta)=\alpha f(\beta)$ $+\beta f(\alpha)=0+0=0$, so $f$ is trivial when restricted to $\mathfrak{b}$ which is in $\mathfrak{a}^{2}$. Thus from the exact sequence (Theorem 2)

$$
0 \rightarrow \mathcal{E}^{1}(R, k) \rightarrow \mathcal{E}^{1}(k[X], k) \rightarrow \operatorname{Hom}_{k[X]}(\mathfrak{b}, k) \rightarrow 0
$$

we get that

$$
0=\operatorname{Hom}_{k[\mathbf{x}]}(\mathfrak{b}, k)=\operatorname{Hom}_{k}(\mathfrak{b} /(\mathfrak{a} \cdot \mathfrak{b}), k) .
$$

Hence $\mathfrak{b} /(\mathfrak{a} \cdot \mathfrak{b})=0$ or $\mathfrak{b}=\mathfrak{a} \cdot \mathfrak{b}$. This gives by induction that $\mathfrak{b}=\mathfrak{a}^{n} \cdot \mathfrak{b}$ for all $n$. Thus every polynomial in $\mathfrak{b}$ has degree greater than $n$ for all $n$. Hence $\mathfrak{b}=0$ and $k[X] \simeq R$.

We refer to [8] for quotient rings.

THEOREM 15. Let $R$ be a polynomial ring and $S$ be a multiplicatively closed subset of $R$ (with $1 \in S, 0 \notin S)$. Let $A$ be the quotient ring $R_{S}$. Then $\mathcal{E}^{2}(A, E)=0$ for all unitary $A$-modules $E$.

Proof. Let $f \in Z^{2}(A, E)$. The restriction of $f$ to $R$ is in $B^{2}(R, E)$. By extending the appropriate map from $R$ to $A$ and taking $f$ minus this extension, we may assume (for the purpose of proving $f \in B^{2}(A, E)$ ) that the restriction of $f$ to $R$ is zero. Thus if $\gamma \in R, \delta \in S$,

$$
f(\gamma \delta, 1 / \delta)=\gamma f(\delta, 1 / \delta)+f(\gamma, 1)-(1 / \delta) f(\gamma, \delta)=\gamma f(\delta, 1 / \delta) .
$$

For $\alpha \in R, \beta \in S$ we write $t(\alpha, \beta)$ for $(1 / \beta) f(\alpha / \beta, \beta)$. Then if $\delta \in S$, 


$$
\begin{aligned}
t(\alpha \delta, \beta \delta) & =(1 / \beta \delta) f(\alpha / \beta, \beta \delta) \\
& =(1 / \beta)[f(\alpha / \beta, \beta)-f(\alpha \delta, 1 / \delta)+(\alpha / \beta) f(\beta \delta, 1 / \delta)]=t(\alpha, \beta) .
\end{aligned}
$$

Thus if $\alpha / \beta=\alpha^{\prime} / \beta^{\prime}$ with $\beta, \beta^{\prime} \in S$, then

$$
t(\alpha, \beta)=t\left(\alpha \beta^{\prime}, \beta \beta^{\prime}\right)=t\left(\alpha^{\prime} \beta, \beta \beta^{\prime}\right)=t\left(\alpha^{\prime}, \beta^{\prime}\right) .
$$

This means that we can write $t(\alpha / \beta)$ for $t(\alpha, \beta)$. It is easily shown that $t$ is linear from $A$ to $E$. Also it is easily computed that $t(\alpha / \beta)=\alpha t(1 / \beta)-f(\alpha, 1 / \beta)$. Thus for $\alpha, \alpha^{\prime} \in R, \beta, \beta^{\prime} \in S$,

$$
\begin{aligned}
&(\alpha / \beta) t\left(\alpha^{\prime} / \beta^{\prime}\right)-t\left(\alpha \alpha^{\prime} / \beta \beta^{\prime}\right)+\left(\alpha^{\prime} / \beta^{\prime}\right) t(\alpha / \beta) \\
&= \alpha \alpha^{\prime} t\left(1 / \beta \beta^{\prime}\right)-(\alpha / \beta) f\left(\beta \alpha^{\prime}, 1 / \beta \beta^{\prime}\right) \\
&-\alpha \alpha^{\prime} t\left(1 / \beta \beta^{\prime}\right)+f\left(\alpha \alpha^{\prime}, 1 / \beta \beta^{\prime}\right)+\left(\alpha^{\prime} / \beta^{\prime}\right) t(\alpha / \beta) \\
&= f\left(\alpha / \beta, \alpha^{\prime} / \beta^{\prime}\right)-\left(1 / \beta \beta^{\prime}\right) f\left(\alpha / \beta, \beta \alpha^{\prime}\right) \\
&+\left(\alpha^{\prime} / \beta \beta^{\prime}\right) f(\alpha / \beta, \beta)=f\left(\alpha / \beta, \alpha^{\prime} / \beta^{\prime}\right) \\
&-\left(1 / \beta \beta^{\prime}\right) f\left(\alpha, \alpha^{\prime}\right)+\left(1 / \beta \beta^{\prime}\right)(\alpha / \beta) f\left(\beta, \alpha^{\prime}\right) \\
&= f\left(\alpha / \beta, \alpha^{\prime} / \beta^{\prime}\right) .
\end{aligned}
$$

Hence $f \in B^{2}(A, E)$.

TheORem 16. Let $A_{S}$ be a quotient ring of a commutative algebra $A$, and let

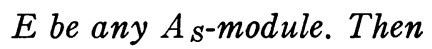

$$
\mathcal{E}^{2}\left(A_{S}, E\right) \simeq \mathcal{E}^{2}(A, E) .
$$

Proof. Let $X$ be a set of indeterminants in one-one correspondence with a generating set for $A$, and let $\phi$ be the natural algebra homomorphism from $k[X]=R$ onto $A$. Let $T=\phi^{-1}(S), \mathfrak{a}$ be the kernel of $\phi$, and $\mathfrak{b}$ be the set of $\beta \in R$ such that $\beta \gamma \in \mathfrak{a}$ for some $\gamma \in T$. Then we have the following commutative, exact diagram:

$$
\begin{aligned}
& 0 \rightarrow \mathfrak{b} R_{T} \rightarrow R_{T} \rightarrow A_{S} \rightarrow 0 \\
& \uparrow \uparrow \uparrow \\
& 0 \rightarrow \mathfrak{a} \rightarrow R \rightarrow A \rightarrow 0 .
\end{aligned}
$$

Now using Theorems 2, 15, and 16 we get

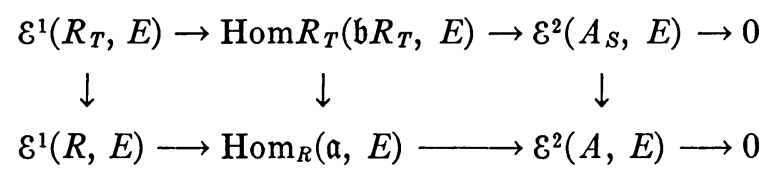

which is easily checked to be commutative. Using the division formula for derivations and some straightforward computations, it is easily checked that 
the first two vertical arrows are isomorphisms, from which it follows that the third is.

Theorem 17. Let $k$ be a perfect field, and let the algebra $A$ over $k$ also be a field. Then $\mathcal{E}^{2}(A, E)=0$ for any unitary $A$-module $E$.

Proof. Let $f \in \mathcal{E}^{2}(A, E)$ be chosen according to the remark after Theorem 5. Let $B$ be the direct sum of $A$ and $E$ with $(\alpha, a)(\beta, b)$ defined as $(\alpha \beta, \alpha b+\beta a+f(\alpha, \beta))$ for $\alpha, \beta \in A, a, b \in E$. Then $B$ is easily checked to be a commutative algebra whose only maximal ideal is $0 \oplus E=M$. Thus $M^{2}=0$ which implies that $B$ is a complete generalized local ring. $k \oplus 0$ is a subfield of $B$ and if $k$ is of characteristic $p, k \oplus 0=k^{p} \oplus 0 \subset B^{p}$. Hence by the proof of the existence of coefficient fields (see [10]), $k \oplus 0$ is contained in a field $D$ with $D \oplus M=B$. For each $\alpha \in A$ there is a unique $(\alpha, g(\alpha)) \in D$. It is easily checked that

$$
f(\alpha, \beta)=-\alpha g(\beta)+g(\alpha \beta)-\beta g(\alpha) \quad \text { for } \alpha, \beta \in A .
$$

Since every algebra is a factor ring of a polynomial algebra, our next theorem gives a calculation of $\mathcal{E}^{2}$ for the irreducible modules.

THEOREM 18. Let $R$ be an algebra over a perfect field $k$, and let $M$ be a maximal ideal which contains an ideal a. If $\mathcal{E}^{2}(R, R / M)=0$, then

$$
\mathcal{E}^{2}(R / \mathfrak{a}, R / M) \simeq\left(\left(M^{2} \cap \mathfrak{a}\right) / M \cdot \mathfrak{a}\right)^{\sharp}
$$

where \# denotes the dual as an $R / M$ vector space.

Proof. Using Theorem 17 and the easily established fact that $\operatorname{Hom}_{R}(\mathfrak{a}, R / M)=\operatorname{Hom}_{R}(\mathfrak{a} / M \cdot \mathfrak{a}, R / M)$, we have the exact diagram

$$
\begin{aligned}
& \mathcal{E}^{1}\left(R / M^{2}, R / M\right) \rightarrow \operatorname{Hom}_{R / M^{2}}\left(M / M^{2}, R / M\right) \rightarrow 0 \\
& \downarrow \downarrow \\
& \mathcal{E}^{1}(R, R / M) \longrightarrow \operatorname{Hom}_{R}(\mathfrak{a} / M \cdot \mathfrak{a}, R / M) \longrightarrow \mathcal{E}^{2}(R / \mathfrak{a}, R / M) \rightarrow 0 .
\end{aligned}
$$

It is easily shown that the first vertical arrow is an isomorphism. With this and vector space theory, the result can now be read from the diagram.

4. Rings of algebraic geometry. In this section we give characterizations, in terms of $\mathcal{E}^{2}$, of regular local rings, of complete intersections, and of nonsingular affine rings.

THEOREM 19. Let the algebra $R$ over a perfect field $k$ be a local ring with maximal ideal $M$. Then $R$ is regular if and only if $\varepsilon^{2}(R, R / M)=0$.

Proof. Suppose that $R$ is regular. Then $M$ has a basis $\alpha_{1}, \alpha_{2}, \cdots, \alpha_{n}$ where $n$ is the Krull dimension of $R$. We prove that $\varepsilon^{2}(R, R / M)=0$ by induction on $n$. For $n=0$ this is merely Theorem 17 . For $n>0$ we consider the exact sequence 


$$
\mathcal{E}^{2}\left(R /\left(\alpha_{n}\right), R / M\right) \rightarrow \varepsilon^{2}(R, R / M) \rightarrow \operatorname{Ext}_{R}^{1}\left(\left(\alpha_{n}\right), R / M\right)
$$

of Theorem 2. Since $R$ is a domain, $\left(\alpha_{n}\right) \simeq R$ and thus the last term of the sequence is zero. Since $R /\left(\alpha_{n}\right)$ is a regular local ring of dimension $n-1$ (see p. 301 of [9]), the first term is zero by induction. Hence $\mathcal{E}^{2}(R, R / M)=0$.

Now suppose that $R$ is a local ring with $\varepsilon^{2}(R, R / M)=0$. We shall prove that $R$ is regular by showing that the associated graded ring $R / M \oplus M / M^{2}$ $\oplus$... is a polynomial ring (see p. 301 of [9]). This in turn will be shown by establishing that each $M^{i} / M^{i+1}$ has the proper dimension over $R / M$. Let $n$ be the dimension of $M / M^{2}$. Let $S$ be the polynomial ring $(R / M)\left[X_{1}, \cdots, X_{n}\right]$ and let $\mathfrak{a}=\left(X_{1}, \cdots, X_{n}\right)$. We now prove by induction on $i$ that $S / \mathfrak{a}^{i}$ is isomorphic to $R / M^{i}$ for all $i$. Suppose $S / \mathfrak{a}^{i}$ is isomorphic to $R / M^{i}$. Since $R / M^{i+1}$ is a complete local ring, by the Cohen structure theorem (see [9]) it will be isomorphic to $S / \mathfrak{a}^{i+1}$ if and only if $M^{i} / M^{i+1}$ and $\mathfrak{a}^{i} / \mathfrak{a}^{i+1}$ have the same dimension. This fact is obvious if $i=1$ and if $i=2$ we may use Theorem 18 to get

$$
\left(M^{i} / M^{i+1}\right)^{\sharp} \simeq \varepsilon^{2}\left(R / M^{i}, R / M\right) \simeq \varepsilon^{2}\left(S / \mathfrak{a}^{i}, R / M\right) \simeq\left(\mathfrak{a}^{i} / \mathfrak{a}^{i+1}\right)^{\dagger} .
$$

COROLlaRy 20. If $\mathfrak{p}$ is a prime in an affine ring $R$ over a perfect field $k$, then $\mathfrak{p}$ will represent a simple point if and only if $\mathcal{E}^{2}(R, Q)=0$ where $Q$ is the quotient field of $R / p$.

COROLLARY 21. Let $N$ be a commutative radical algebra (in the sense of Jacobson) which satisfies the ascending chain condition on ideals. Then there will be a symmetric inner product with $(\alpha, \beta \gamma)=(\alpha \beta, \gamma)$ for $\alpha, \beta, \gamma \in N$ and with the inner product not of the form $(\alpha, \beta)=g(\alpha \beta)$ for a functional $g$, if and only if $N^{*}$ is not a regular local ring.

Proof. The details, such as that $N^{*}$ will be local (in particular Noetherian) are left to the reader.

THEOREM 22. Let $R$ be an affine ring (that is, a finitely generated algebra with identity) over a perfect field $k$. Then $R$ will be nonsingular if and only if $\mathcal{E}^{2}(R, E)=0$ for every finitely generated unitary $R$-module $E\left(^{4}\right)$.

Proof. Suppose $\varepsilon^{2}(R, E)=0$ for all finitely generated $E$. Then if $M$ is a maximal ideal, $\mathcal{E}^{2}(R, R / M)=0$; thus by Corollary $20, R_{M}$ is regular. Since any of the local rings of $R$ can be gotten by going to some $R_{M}$ first, it follows from p. 307 of [9] that $R$ is nonsingular.

(4) The methods of proof here show that if $A$ is a regular local ring of an affine ring, then $\mathcal{E}^{2}(A, E)=0$ for all finitely generated $E$. If in addition, $A$ is the local ring at a zero dimensional point and $k$ is algebraically closed of characteristic not 2 or 3 , then $\mathcal{E}^{3}(A, E)=0$ for all such $E$. This is proved by using that $A=M^{*}$, that $\operatorname{dim} H^{3}(M, k)=\left({ }_{3}^{n}\right)$, that $\delta\left(C^{2}(M, k)\right)$ is in the kernel of the alternating map $T$, and that $C^{3}\left(M / M^{2}, k\right)$ modulo the image of $T$ already has dimension at least $\left(\begin{array}{l}n \\ 3\end{array}\right) . \mathcal{E}^{3}(A, k)=0$ implies $\mathcal{E}^{3}(A, E)=0$ as below except: $A \otimes A$ is Noetherian since $(R \otimes R)_{S \otimes S} \simeq$ $R_{S} \otimes R_{S}$, and $\mathcal{E}^{3}(A, E)$ is a submodule of $H^{3}(A, E)$ by a use of the characteristic of $k$ not being 2 . 
Now suppose $R$ is nonsingular, and just suppose that there is a finitely generated $E$ with $\varepsilon^{2}(R, E) \neq 0$. Let $L$ be maximal in $E$ with the property that $\varepsilon^{2}(R, E / L) \neq 0$. If $M$ is any maximal ideal, we have the exact sequence

$$
\mathcal{E}^{2}(R, L: M / L) \rightarrow \mathcal{E}^{2}(R, E / L) \rightarrow \mathcal{E}^{2}(R, E / L: M)
$$

where $L: M / L$ is a direct sum of copies of $R / M$. Thus with Corollary 20 and the assumption that $R$ is nonsingular, it is easily shown that $\mathcal{E}^{2}(R, L: M / L)=0$ (the reader can either show that $\mathcal{E}^{2}$ preserves direct sums or else use Theorem 1 a finite number of times). By the choice of $L$, this means that $L=L: M$. Hence $M$ is not in any of the primes of $L$; thus it is not in the union of these primes (see [8]); and thus there is an $\alpha \in M$ with $\alpha$ not in any of the primes of $L$. This means that $L: \alpha=L$, and in particular, it means that $L \neq \alpha E+L$. Thus

$$
0 \rightarrow E / L \stackrel{\alpha}{\rightarrow} E / L \rightarrow E /(\alpha E+L) \rightarrow 0
$$

gives

$$
\mathcal{E}^{2}(R, E / L) \stackrel{\alpha}{\rightarrow} \mathcal{E}^{2}(R, E / L) \rightarrow 0 .
$$

We have proven for every maximal ideal $M$, that $M \cdot \mathcal{E}^{2}(R, E / L)=\mathcal{E}^{2}(R, E / L)$. It is an easy consequence of the intersection theorem that this implies a contradiction, once we have that $\mathcal{E}^{2}(R, E / L)$ is finitely generated. Now $R$ is affine; thus $R \otimes R$ is also. This implies that $R \otimes R$ is Noetherian. There exists an ideal $\mathfrak{a}$ with $R \otimes R / \mathfrak{a}$ isomorphic to $R$; thus both $R$ and $E / L$ are finitely generated as $R \otimes R$-modules. This implies that $\operatorname{Ext}_{A \otimes A}^{2}(A, E / L)$ is finitely generated as an $A \otimes A$-module, and thus as an $A$-module (see p. 122 of [3]). But

$$
\mathcal{E}^{2}(A, E / L) \subset H^{2}(A, E / L) \simeq \operatorname{Ext}_{A \otimes A}^{2}(A, E / L) .
$$

CoRollary 23. Let $\mathfrak{a}$ be an ideal in a nonsingular affine ring $R$. Then $R / \mathfrak{a}$ will be nonsingular if and only if there is an algebra homomorphism from $R / \mathfrak{a}$ to $R / \mathfrak{a}^{2}$ which when followed by the canonical map gives the identity.

Proof. This is equivalent to there being a derivation from $R / \mathfrak{a}^{2}$ to $\mathfrak{a} / \mathfrak{a}^{2}$ which is the identity on $\mathfrak{a} / \mathfrak{a}^{2}$ (take the identity minus the homomorphism). The exact sequence

$$
\mathcal{E}^{1}(R, E) \rightarrow \operatorname{Hom}_{R}(\mathfrak{a}, E) \rightarrow \mathcal{E}^{2}(R / \mathfrak{a}, E) \rightarrow 0
$$

gives the singularity of $R / \mathfrak{a}$ in terms of the raising of homomorphisms to derivations. The corollary can now be reasoned out from this sequence.

TheOREM 24. Let $R$ with maximal ideal $M$, be a local ring (over a perfect field $k$ ) which is a factor ring of some regular local ring (say $R$ is a local 
ring of algebraic geometry). Let $\operatorname{dim} R=n$ and $\operatorname{dim}_{R / M}\left(M / M^{2}\right)=m$. Then $\operatorname{dim}_{R / M} \mathcal{E}^{2}(R, R / M) \geqq m-n$ with equality holding if and only if $R$ is a complete intersection.

Proof. Suppose $A$, with maximal ideal $N$, is a regular local ring with $A / \mathfrak{a}$ isomorphic to $R$. Then by straightforward usual arguments, a new $A$ can be chosen with $\mathfrak{a} \subset N^{2}$. Thus by Theorem 18 and duality theory for vector spaces

$$
\operatorname{dim}_{R / M} \mathcal{E}^{2}(R, R / M)=\operatorname{dim}_{A / N}(\mathfrak{a} / N \cdot \mathfrak{a}) .
$$

Since $\operatorname{dim} A=m$ and $\operatorname{dim} \mathfrak{a}=n$, the result now follows from the Krull dimension theorem.

Corollary 25. Let $A$ be an affine ring of dimension $r$ over a perfect field $k$. Let $\mathfrak{p}$ be a prime of $A$ and let $Q$ be the quotient field of $A / \mathfrak{p}$. Then

$$
\operatorname{dim}_{Q} \mathcal{E}^{1}(A, Q)-\operatorname{dim}_{Q} \mathcal{E}^{2}(A, Q) \leqq r
$$

with equality holding if and only if $\mathfrak{p}$ represents a complete intersection.

Proof. It can be checked that

$$
\mathcal{E}^{1}(A, Q) \simeq \mathcal{E}^{1}\left(A_{\mathfrak{p}}, Q\right) \simeq\left(\mathfrak{p} \cdot A_{\mathfrak{p}} / \mathfrak{p}^{2} \cdot A_{p}\right)^{\sharp} \oplus \mathcal{E}^{1}(Q, Q)
$$

and that $\operatorname{dim}_{Q} \mathcal{E}^{1}(Q, Q)=\operatorname{dim} \mathfrak{p}$.

Added in proof. My student M. Barr has pointed out that "finitely generated" may be removed from the statement of Theorem 22 by using the device of Corollary 23.

\section{BIBLIOGRAPHY} 55-106.

1. S. Eilenberg and S. Mac Lane, On the groups $H(\pi, n)$. I, Ann. of Math. (2) 58 (1953),

2. G. Hochschild, On the cohomology groups of an associative algebra, Ann. of Math. (2) 46 (1945), 58-67.

3. H. Cartan and S. Eilenberg, Homological algebra, Princeton Univ. Press, Princeton, N. J., 1956.

4. G. Hochschild, On the cohomology theory for associative algebras, Ann. of Math. (2) 47 (1946), 568-570.

5. - Cohomology and representations of associative algebras, Duke Math. J. 14 (1947), 921-948.

6. S. Eilenberg and S. Mac Lane, Cohomology theory in abstract groups. II, Ann. of Math. (2) 48 (1947), 326-341.

7. S. Eilenberg and T. Nakayama, On the dimension of modules and algebras. II, Nagoya Math. J. 9 (1955), 1-16.

8. O. Zariski and P. Samuel, Commutative algebra, Vol. I, Van Nostrand, Princeton, N. J., 1958.

9. Commutative algebra, Vol. II, Van Nostrand, Princeton, N. J., 1960.

10. M. Narita, On the structure of complete local rings, Proc. Internat. Sympos. on Algebraic Numbers (Tokyo, 1955), pp. 251-253.

University of Pennsylvania, Philadelphia, Pennsylvania 